\title{
Sublaminar bands in oncological spine surgery: illustrative cases
}

\author{
Godard C. W. de Ruiter, MD, PhD, ${ }^{1}$ Valerio Pipola, $\mathrm{MD},{ }^{2}$ Cristiana Griffoni, $\mathrm{PhD},{ }^{2}$ and Alessandro Gasbarrini, $\mathrm{MD}^{2}$ \\ ${ }^{1}$ Department of Neurosurgery, Haaglanden Medisch Centrum, The Hague, The Netherlands; and ${ }^{2}$ Department of Spine Surgery, IRCCS Istituto Ortopedico Rizzoli, Bologna, \\ Italy
}

\begin{abstract}
BACKGROUND Sublaminar bands have been used in addition to pedicle screw placement in the correction of idiopathic scoliosis forming a so-called hybrid construct.

OBSERVATIONS In this article, the authors present several cases that demonstrate the potential applications of sublaminar bands in oncological spine surgery. The potential applications are divided into three categories: (1) as an additional tool in salvage procedures, (2) to correct kyphosis in pathological fractures, and (3) for bone graft anchoring to the spine.
\end{abstract}

LESSONS The cases presented in this article demonstrate the potential beneficial effects of the sublaminar bands in addition to pedicle screw placement. https://thejns.org/doi/abs/10.3171/CASE21111

KEYWORDS sublaminar bands; hybrid construct; pathological kyphosis correction; roof technique; posterior column reconstruction

Sublaminar bands have been used for over a decade now in the surgical correction of thoracolumbar scoliosis as an additive to pedicle screw fixation forming a so-called hybrid construct. ${ }^{1,2}$ The rationale for the use of these hybrid constructs in scoliosis is that they better preserve the physiological sagittal profile than constructs that merely consist of pedicle screws, ${ }^{3}$ theoretically reducing the risk of proximal junctional kyphosis. ${ }^{4}$ There have been only a few reports on the use of sublaminar bands in the surgical treatment of degenerative spinal disease, vertebral trau$\mathrm{ma}^{5-7}$ and more recently in the cervical spine ${ }^{8}$ and at the cervicothoracic junction. ${ }^{9,10}$

In the present article, the authors present the potential applications of sublaminar bands in oncological spine surgery using several illustrative cases. The different indications that are discussed are subdivided into three categories: (1) as a salvage procedure at levels at which there is no option to place a pedicle screw, (2) as an additional tool for kyphosis reduction in pathological vertebral fractures, and (3) for the anchoring of a bone graft. As we are aware that not all readers may be familiar with the principles of sublaminar bands, additional information is first provided on sublaminar bands.
Currently, there are several sublaminar bands from different companies available off the shelf. In the present study, polyester sublaminar bands (Jazz bands, Implanet) have been used. The tip of the band contains a small segment of metal that can be bent into a small U-shape to tunnel the band from one interlaminar space under the lamina to the other interlaminar space. The end of the tunneled band is subsequently inserted through the buckle at the other end of the band, thereby creating a loop. Before the loop is closed, the band is inserted through a titanium connector that can be placed anywhere along a rod and is fixed (loosely) with a small screw. The loop is subsequently placed around a reduction tool. Gradually increasing force can be applied on the band using this tool by turning the handle in a clockwise fashion. This maneuver can be performed on a rod that is already fixed at both ends in the tulip heads of pedicle screws (to pull the lamina toward the rod), but also on a rod that is only fixed at one end. The latter technique can be useful, for example, when correcting a kyphotic deformity. The force that is applied on the rod and screws can thereby be divided over multiple levels if pedicle screws with extended tulip heads are used in which the end caps are tightened gradually while the force on the bands is increased. When enough force is applied, 
the band can be locked by tightening the screw that was first loosely placed inside the connector. Finally, the remaining band is removed by cutting the loop about $1 \mathrm{~cm}$ outside the connector.

\section{Illustrative Cases \\ Salvage Procedure}

One potential application of sublaminar bands is as a salvage procedure, when it is not possible to place a pedicle screw. In thoracolumbar scoliosis surgery, this may sometimes be the case, for example, if the size of the pedicle is too small. In oncological spine surgery, there may be additional reasons to place sublaminar bands at a level, such as when it is not possible to place a screw in the pedicle because of breakage of a previously placed screw (case 1) or placement of vertebral body stents (case 2).

\section{Case 1}

A 53-year-old female was referred to the Instituto Ortopedico Rizzoli because of the failure of a construct (breakage of pedicle screws in $L 5$ on the right) that had been placed in another hospital 4 years earlier (Fig. $1 A$ and $B$ ). The indication for the initial surgery was a recurrent metastasis of $L 4$ from a renal cell carcinoma. The primary tumor had been treated with left nephrectomy and adjuvant radiotherapy. She had symptoms of low back pain without any motor or sensory deficit. The construct was revised by placing additional pedicle screws in L3 and S1. The broken screw in L5 on the
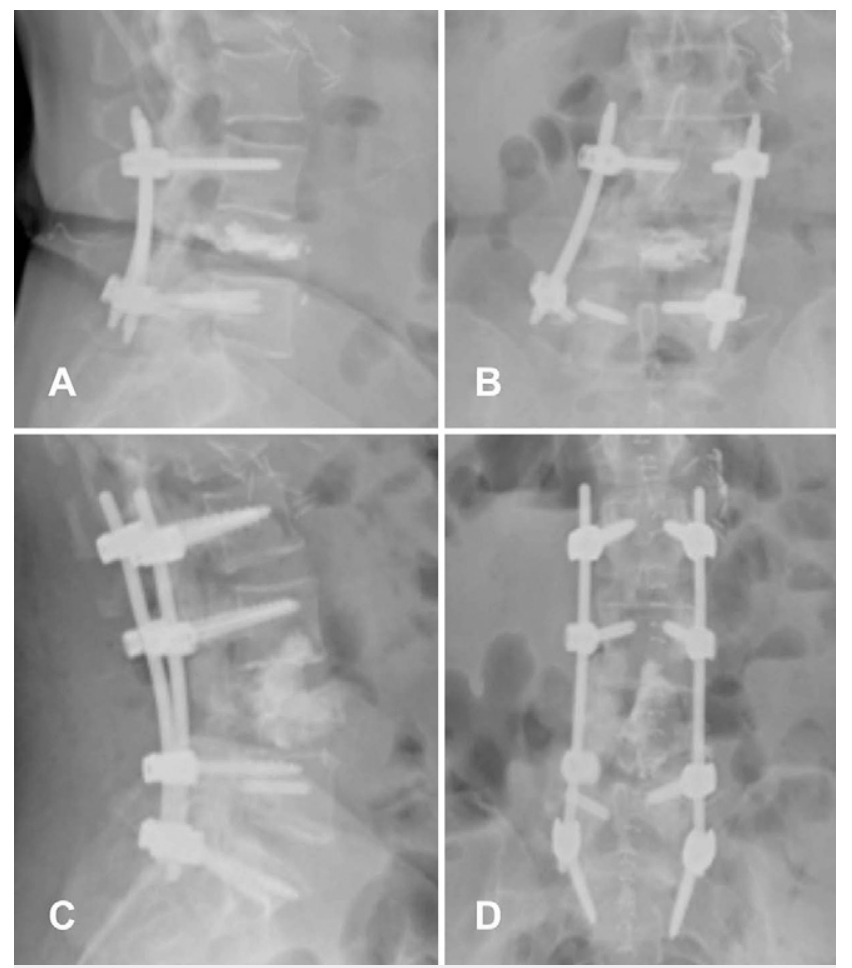

FIG. 1. Preoperative ( $A$ and $B$ ) and postoperative ( $C$ and $D)$ radiographs of case 1 showing potential application of a sublaminar band as a salvage procedure when placement of a pedicle screw is not possible because of breakage of the previously placed screw inside the pedicle of $L 5$ on the right side. right side could not be removed; therefore, a sublaminar band was used in this case to strengthen the construct at the previous site of screw breakage (Fig. 1C and D). The postoperative course was complicated by a wound infection for which she needed revision surgery. There was no complication or revision required of the sublaminar band. Six months after the surgery, the patient was free of pain, the wound was completely closed, and there was no failure of the construct.

\section{Case 2}

A 77-year-old man who had been treated elsewhere with radiotherapy for a lumbar lymphoma was referred to the Haaglanden Medical Center because of severe pain while he was in the vertical position. He received high doses of morphine. A computed tomography $(C T)$ scan showed destruction of the $L 4$ and $L 5$ vertebral bodies with a vacuum phenomenon in the L4-5 intervertebral disc space (Fig. 2A). Intraoperatively, the lumbar spine was fixed by placing pedicle screws at the levels of $\mathrm{L} 3$ and $\mathrm{S} 1$. Vertebral body stents (SpineJack, Stryker) were placed in the L4 and L5 corpora through the pedicles bilaterally. After augmentation of the stents, cement was introduced through the same trajectory. At the levels of $\mathrm{L} 4$ and $\mathrm{L} 5$, sublaminar bands were inserted at both sides and were connected to the rods (Fig. 2B). Postoperatively, the patient's pain symptoms significantly improved. Two years after the surgery, he was free of morphine, and the construct was still stable/unchanged compared with directly postoperatively.

\section{Correction of Kyphosis}

Case 3

A 68-year-old man was referred to the Haaglanden Medical Center with a solitary spinal metastasis of $T 12$ and collapse of the L1 vertebral body. He experienced severe pain in the standing position. Although he had no neurological deficit, an epidural tumor at the T12 level was compressing the cord/conus (Fig. $3 \mathrm{~A}$ and $\mathrm{B}$ ). He had no previous history of malignancy. CT of the thorax/abdomen showed a lung tumor with metastases in the liver and both kidneys. Surgery was performed to decompress the spinal cord by T12 laminectomy and to fix the thoracolumbar junction.
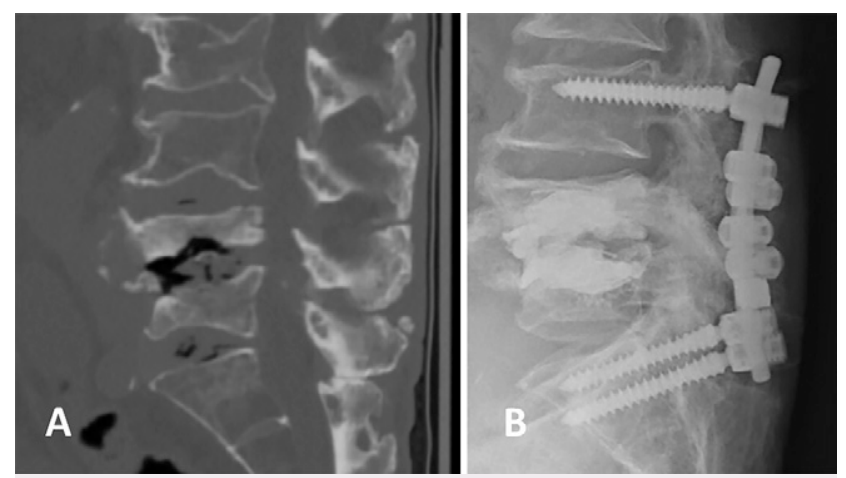

FIG. 2. A: Preoperative sagittal CT image of case 2 demonstrating spinal instability after radiotherapy for lumbar lymphoma. B: Postoperative radiograph in standing position shows that a stable hybrid construct was achieved with placement of sublaminar bands at the levels at which vertebral body stents had been placed. 

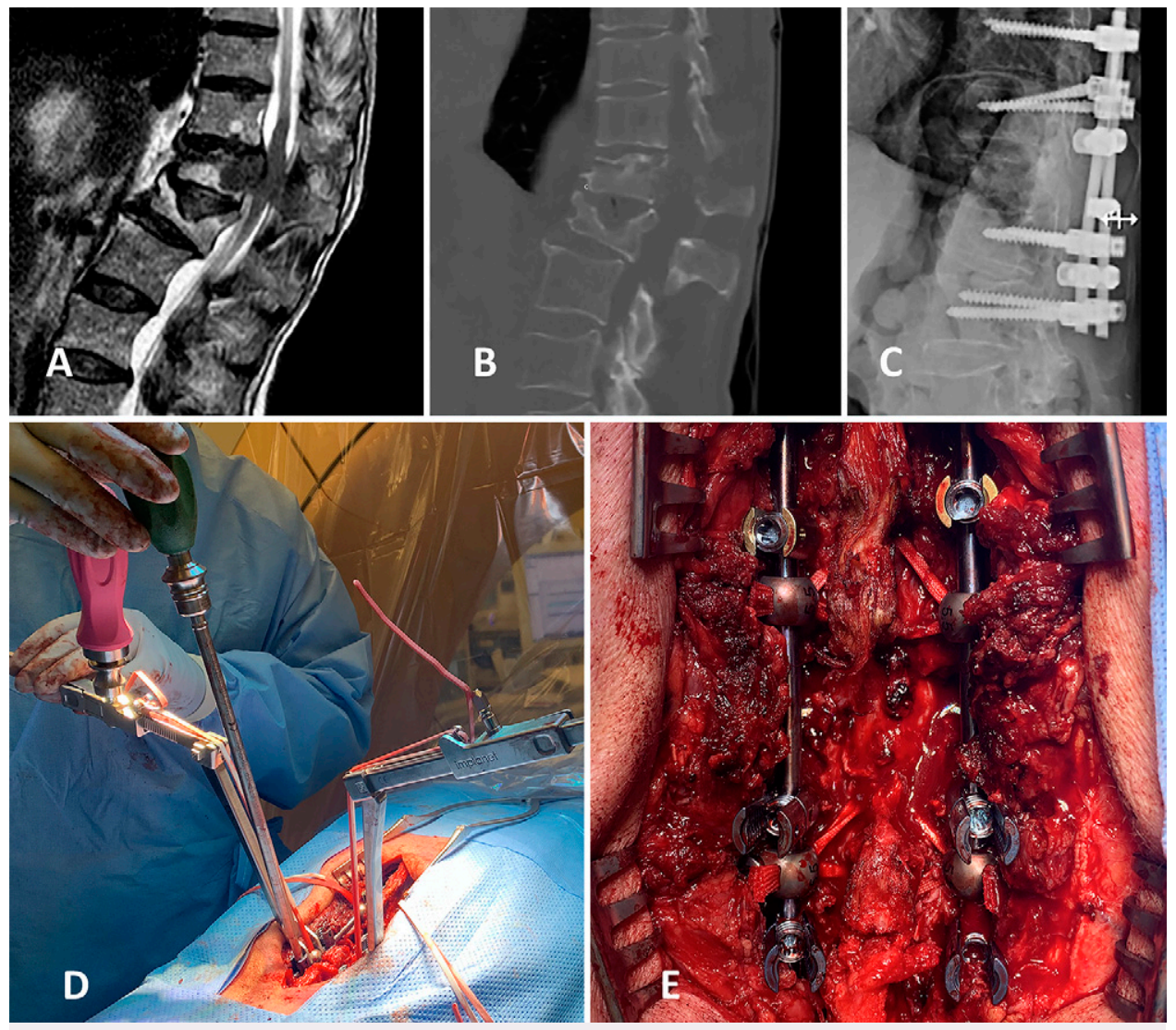

FIG. 3. Preoperative sagittal T2-weighted magnetic resonance imaging (A) and CT (B) scans of case 3 showing how kyphosis was corrected using a hybrid construct (C; postoperative standing radiograph). Intraoperative photographs show the technique (D) by which the forces during correction are distributed over the extended-head pedicle screws and sublaminar bands by alternative tightening of the set screws (screwdriver with green handle) and sublaminar bands (pink handle) and the final result (E) with pedicle screws and sublaminar bands connected to the same rods on both sides.

The spine was fixed from $T 10$ to $L 2$ by placement of pedicle screws at the levels of $T 10, T 11, L 1$, and L2. In addition, sublaminar bands were placed around the lamina at T11 and L1. At the level of T11, they were placed because of poor bone quality to prevent screw pullout, and at the L1 level additionally for the correction of the kyphosis. Before correction, the end caps in the tulip heads at T10 and T11 and the sublaminar bands at lamina T11 were tightened. Reduction was subsequently performed over straight rods placed in the extended tulip heads by gradually increasing the tension on the bands around the lamina at L1 on both sides and simultaneously lowering the end caps in the extended tulip heads of the pedicle screws at L1 and L2 (Fig. 3D). This procedure was done alternatively on both sides to distribute the total force over the four pedicle screws and the lamina at L1 through the two sublaminar bands (Fig. 3D). The intraoperative result is demonstrated in Fig. $3 \mathrm{E}$. The standing radiograph (Fig. $3 \mathrm{C}$ ), which was obtained 1 day after the surgery, when the patient was again able to mobilize with reduced pain compared with preoperatively, showed a clear reduction of the kyphosis angle compared with the preoperatively obtained magnetic resonance imaging and CT scans (Fig. $3 \mathrm{~A}$ and B), which had been obtained in the horizontal position. There were no postoperative complications, but the patient died several months after the surgery because of progression of disease.

\section{The Roof Technique \\ Case 4}

A 14-year-old boy was referred to the Instituto Ortopedico Rizzoli with a chondroblastoma-like osteosarcoma arising from T12 and characterized by an epidural involvement at the same level with an extracompartmental mass extending from T11 and L2 (Fig. 4A). He underwent a T12 intralesional resection with an en bloc excision of the posterior arches from T11 to L2. Anterior column reconstruction was achieved with an allograft connected to posterior carbon fiber-reinforced polyetheretherketone instrumentation with pedicle screws (Fig. $4 B$ and $C)$. The posterior column instead was reconstructed with a shaped allograft anchored to posterior instrumentation with sublaminar bands that were passed around the rods and not under the laminae: the so-called roof technique (Fig. 4D). A CT scan at the 16-month follow-up after adjuvant chemotherapy and high particle radiation therapy demonstrated implant stability and fusion of both the anterior and posterior allografts (Fig. $4 \mathrm{E}-\mathrm{G}$ ). 

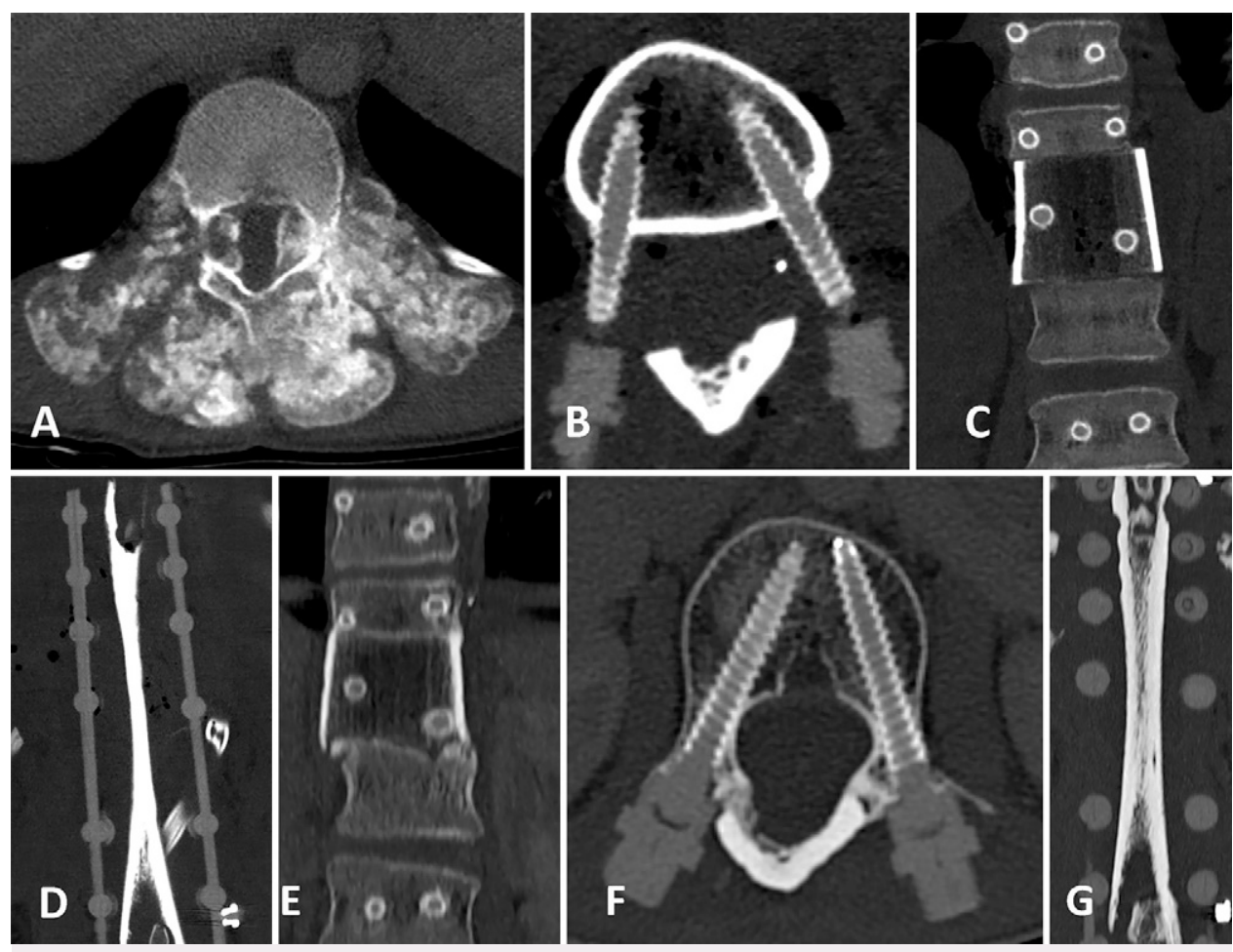

FIG. 4. Preoperative transverse CT image (A) of case 4 showing a chondroblastoma-like osteosarcoma arising from the vertebral body of T12. Postoperative transverse (B) and coronal (C) CT images of the anteriorly placed allograft fixed with two screws and posteriorly placed allograft fixed with sublaminar bands (D). CT images after 16 months of follow-up showing fusion of the anteriorly placed allograft (E) and the posteriorly placed allograft that was fixed with sublaminar bands ( $\mathbf{F}$ and $\mathbf{G})$.

\section{Discussion}

Observations

In this article, we present several cases to illustrate the potential use of sublaminar bands in oncological spine surgery. The different indications were subdivided into three categories that are separately discussed below.

\section{Salvage Procedure}

The first two cases show the potential application of sublaminar bands in patients in whom it is not possible to place pedicle screws at a certain level because of breakage of a previously placed screw (case 1) or because of the placement of vertebral body stents (case 2). Although it would have been possible to place shorter screws and/or place screws in a different direction (e.g., cortical trajectory bone ${ }^{11}$ ), sublaminar bands in these situations may also provide an alternative technique, somewhat similar to the use of laminar hooks. An additional advantage of sublaminar bands is that they provide more stability to the construct because the bands counteract the pullout forces on the pedicle screws, thanks to the connection of the bands around the laminae onto the rods.

\section{Correction of Kyphosis}

An additional advantage of sublaminar bands, which are especially useful in oncological spine surgery, is the potential to correct the sagittal alignment in the case of pathological fractures. Oncological spine surgery nowadays is shifting toward a more elderly population because of both increased general life expectancy and improved survival of cancer patients due to new systematic therapies. While in younger patients corpectomy procedures (piecemeal or en bloc) are often performed to remove tumors and expandable cages are used to reconstruct the spine, ${ }^{12}$ in elderly patients, these procedures are often too extensive and are associated with more intra- and postoperative risks. An important postoperative complication, for example, is the subsidence of the cage due to osteoporotic bone.

Spinal metastases in elderly patients also frequently lead to pathological fractures. In less severe cases of kyphosis due to collapse of the vertebral body, an alternative would be to use cement-augmented pedicle screws, ${ }^{13}$ which have the advantage that both the screws and the vertebral stent can be placed percutaneously, ${ }^{14}$ thus reducing the risk of postoperative infection. In our third case, this was not considered to be an option because of the large Cobb angle, which, in our experience, causes a higher chance of failure of such a construct, and also because of the high load on the thoracolumbar junction. Follow-up in this case was limited because of rapid progression of the cancer, but in the experience of the first author, the application of the same technique in the treatment of osteoporotic vertebral body fractures has led to stable constructs with 1 year of follow-up (G.C.W. de Ruiter, unpublished data, 2020). 


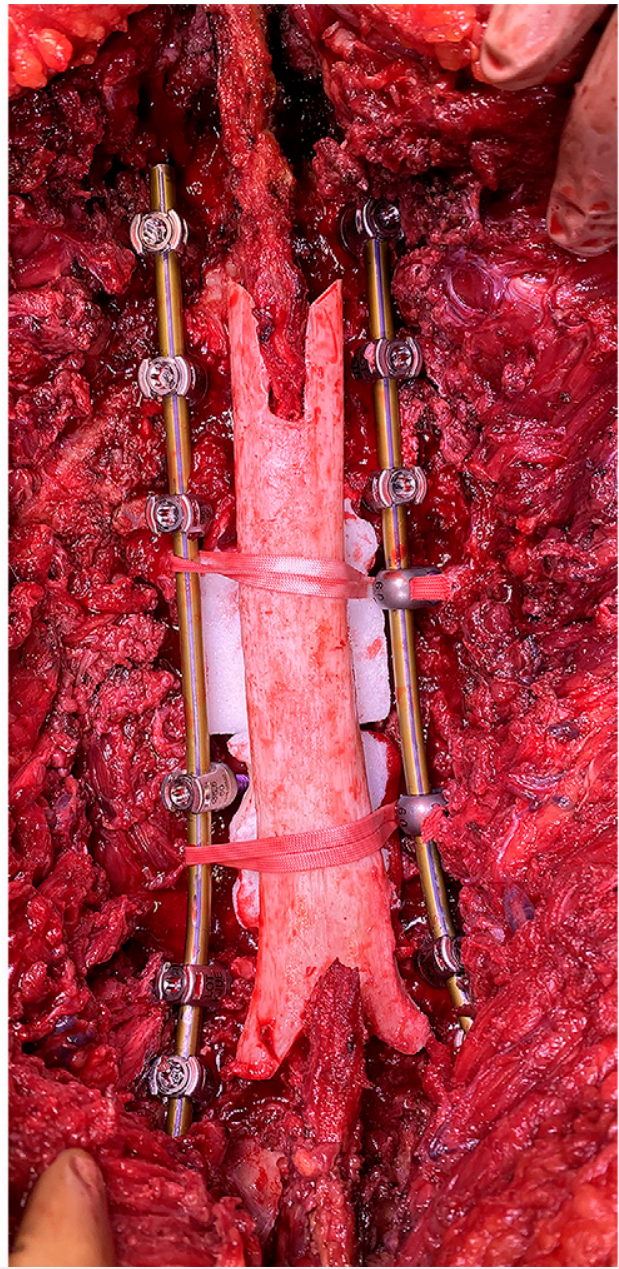

FIG. 5. Intraoperative image of the roof technique. A posteriorly placed allograft is fixed to the rods with the same Teflon bands used in previous cases to pass under the laminae.

\section{The Roof Technique}

Some papers have investigated reconstructive options for the posterior column in order to increase the stability of anterior column reconstruction and posterior fixation in larger defects in bone due to an en bloc resection. ${ }^{15,16}$ The reconstruction of the posterior column is also useful in the surgery for adult spine deformity requiring corrective osteotomies of Schwab grades $3-6,{ }^{17}$ which, in addition to determining large posterior bone defects, can lead to the development of pseudoarthrosis associated with mechanical failure of posterior instrumentation. The senior author (A.G.) has devised a reconstructive modality of the posterior column, which involves the use of a tibia or femur allograft that is shaped and positioned to straddle the spinous processes proximal and distal to the resection. The graft is anchored to the rods using the sublaminar bands (Fig. 5). This technique is also called the "roof technique." This technique provides a posterior fusion bony surface and protects the spinal cord and the cauda equina from accidental injuries in case of revision surgery. In case of lumbar application, especially after corrective osteotomies, the "roof" gives the construct a delta shape aspect that is associated with a reduction in rod stress. ${ }^{18}$

\section{Lessons}

In this article, several potential applications of sublaminar bands in oncological spine surgery are presented, including (1) in salvage procedures (when it is not possible, for example, to place a pedicle screw), (2) in the correction of kyphosis after a pathological fracture, and (3) to anchor bone graft to a posterior construction (the roof technique). These potential applications of sublaminar bands for oncological spine surgery may, of course, also be used in other areas of spine surgery, such as in the osteoporotic spine.

\section{References}

1. Viswanathan A, Johnson KK, Whitehead WE, et al. Hybrid spinal constructs using sublaminar polyester bands in posterior instrumented fusions in children: a series of 5 cases. Neurosurgery. 2010;66(5):862-867.

2. Ilharreborde $B$, Even J, Lefevre $Y$, et al. Hybrid constructs for tridimensional correction of the thoracic spine in adolescent idiopathic scoliosis: a comparative analysis of universal clamps versus hooks. Spine (Phila Pa 1976). 2010;35(3):306-314.

3. Vora V, Crawford A, Babekhir N, et al. A pedicle screw construct gives an enhanced posterior correction of adolescent idiopathic scoliosis when compared with other constructs: myth or reality. Spine (Phila Pa 1976). 2007;32(17):1869-1874.

4. La Rosa G, Giglio G, Oggiano L. The Universal Clamp hybrid system: a safe technique to correct deformity and restore kyphosis in adolescent idiopathic scoliosis. Eur Spine J. 2013;22(suppl 6):S823-S828.

5. Tegos S, Charitidis C, Korovessis PG. Hybrid circumferential fixation for degenerative lumbosacral spine disease: posterior lumbar interbody fusion plus universal clamp rod-band instrumentation: a novel technique for lumbosacral fixation. Spine (Phila Pa 1976). 2014;39(7): E441-E449.

6. Unterweger MT, Kandziora F, Schnake KJ. Hybrid stabilization of thoracic spine fractures with sublaminar bands and transpedicular screws: description of a surgical alternative and review of the literature. Case Rep Orthop. 2015;2015:857607.

7. Gazzeri R, Faiola A, Galarza M, Tamorri M. Universal Clamp system in thoracolumbar spinal fixation: technical note. Acta Neurochir (Wien). 2009;151(12):1673-1680.

8. Smith LGF, Hoang N, Shaikhouni A, Viljoen S. Sublaminar polyester bands as a salvage fixation method in the cervical spine: novel application in two patients. J Neurosurg Spine. 2019;30(6):729-861.

9. Pipola V, Boriani S, Ghermandi R, et al. Composite PEEK/carbon fiber pre-shaped rods and sublaminar bands for posterior stabilization of cervico-thoracic junction: a novel technique. J Clin Neurosci. 2020;72:429-433.

10. Boriani S, Pipola V, Cecchinato R, et al. Composite PEEK/carbon fiber rods in the treatment for bone tumors of the cervical spine: a case series. Eur Spine J. 2020;29(12):3229-3236.

11. Santoni BG, Hynes RA, McGilvray KC, et al. Cortical bone trajectory for lumbar pedicle screws. Spine J. 2009;9(5):366-373.

12. de Ruiter GC, Lobatto DJ, Wolfs JF, et al. Reconstruction with expandable cages after single- and multilevel corpectomies for spinal metastases: a prospective case series of 60 patients. Spine J. 2014;14(9):2085-2093.

13. Barzilai O, McLaughlin L, Amato MK, et al. Minimal access surgery for spinal metastases: prospective evaluation of a treatment algorithm using patient-reported outcomes. World Neurosurg. 2018;120: e889-e901.

14. Moussazadeh N, Rubin DG, McLaughlin L, et al. Short-segment percutaneous pedicle screw fixation with cement augmentation for tumor-induced spinal instability. Spine J. 2015;15(7):1609-1617.

15. Chung JY, Kim SK, Jung ST, Lee KB. New posterior column reconstruction using titanium lamina mesh after total en bloc spondylectomy of spinal tumour. Int Orthop. 2013;37(3):469-476. 
16. Wiedemayer $H$, Sandalcioglu IE, Aalders $M$, et al. Reconstruction of the laminar roof with miniplates for a posterior approach in intraspinal surgery: technical considerations and critical evaluation of follow-up results. Spine (Phila Pa 1976). 2004;29(16):E333-E342.

17. Schwab F, Blondel B, Chay E, et al. The comprehensive anatomical spinal osteotomy classification. Neurosurgery. 2014;74(1):112-120.

18. Berjano P, Xu M, Damilano M, et al. Supplementary delta-rod configurations provide superior stiffness and reduced rod stress compared to traditional multiple-rod configurations after pedicle subtraction osteotomy: a finite element study. Eur Spine J. 2019;28(9):2198-2207.

\section{Disclosures}

The authors report no conflict of interest concerning the materials or methods used in this study or the findings specified in this paper.

\section{Author Contributions}

Conception and design: de Ruiter, Gasbarrini. Acquisition of data: de Ruiter. Analysis and interpretation of data: de Ruiter, Griffoni. Drafting the article: de Ruiter, Pipola. Critically revising the article: de Ruiter, Griffoni, Gasbarrini. Reviewed submitted version of manuscript: de Ruiter, Griffoni, Gasbarrini. Approved the final version of the manuscript on behalf of all authors: de Ruiter. Administrative/technical/material support: de Ruiter. Study supervision: Gasbarrini.

\section{Correspondence}

Godard C. W. de Ruiter: Haaglanden Medical Center, The Hague, The Netherlands.g.de.ruiter@haaglandenmc.nl. 